\title{
Downregulation of VRK1 reduces the expression of BANF1 and suppresses the proliferative and migratory activity of esophageal cancer cells
}

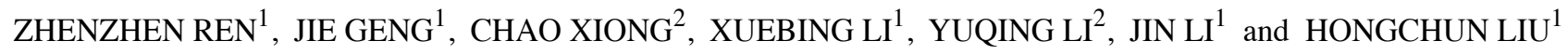 \\ ${ }^{1}$ Department of Medical Laboratory, The First Affiliated Hospital, Zhengzhou University; ${ }^{2}$ Department of Medical Laboratory, \\ The Second Affiliated Hospital of Henan University of Traditional Chinese Medicine, Zhengzhou, Henan 450052, P.R. China
}

Received July 1, 2019; Accepted April 15, 2020

DOI: $10.3892 / \mathrm{ol} .2020 .11654$

\begin{abstract}
Esophageal squamous cell carcinoma (ESCC) is a common malignancy worldwide. The disease has a poor prognosis and a low 5-year survival rate. Therefore, it is necessary to identify new strategies to optimize the treatment of ESCC. Vaccinia-related kinase (VRK1) and barrier-to-autointegration factor 1 (BANF1) are overexpressed in ESCC. In the present study, the roles of $V R K 1$ and $B A N F 1$ were explored in the development of ESCC. In the present study, the effects of small interfering (si)RNA-induced downregulation of VRKI on $B A N F 1$ expression were investigated as well as the effects on proliferative and migratory activity of ESCC cells. Western blot analysis indicated that the protein expression levels of $B A N F 1$ were decreased following siRNA depletion of VRK1. Furthermore, the depletion of VRK1 expression inhibited the proliferation and migration of ESCC cell lines, and flow cytometry analysis indicated that the depletion of VRK1 triggered cell cycle arrest mainly in the $S$ phase. These results suggested that $V R K 1$ and $B A N F 1$ may have pivotal roles in the progression of ESCC.
\end{abstract}

\section{Introduction}

Esophageal cancer is one of the most common malignant tumors and ranks as the sixth leading cause of global mortalities, and the third leading cause of death in China $(1,2)$. Esophageal carcinoma mainly includes esophageal squamous cell carcinoma (ESCC) and esophageal adenocarcinoma; ESCC is the predominant subtype of esophageal cancer in developing countries accounting for $>90 \%$ of all esophageal cancer subtypes in China (3). In 2018, the global cancer

Correspondence to: Dr Hongchun Liu, Department of Medical Laboratory, The First Affiliated Hospital, Zhengzhou University, 1 Jianshe East Road, Zhengzhou, Henan 450052, P.R. China E-mail: xingyunerliu@163.com

Key words: esophageal squamous cell carcinoma, vaccinia-related kinase 1, barrier-to-autointegration factor 1 observatory reported 572,034 new cases of esophageal cancer and 508,585 deaths from esophageal cancer between males and females combined (2). Despite recent advances in surgical and therapeutic techniques, such as chemotherapy and radiotherapy, extensive metastasis has led to a poor 5-year survival rate of $\sim 15-25 \%$ (4). Therefore, there is an urgent need to explore the underlying molecular mechanisms that can be used to improve the diagnosis and treatment of ESCC.

Vaccinia-related kinase (VRK) is a member of the serine/ threonine kinase family in mammals, which performs pivotal functions by regulating a variety of cellular and physiological activities through phosphorylation reactions (5). VRK1 encodes a protein of 396 amino acids in length and was originally discovered from a cDNA library enriched in human fetal-specific liver genes (6). VRK1 is localized to the cell nuclei and is one of the three subtypes of $V R K$; it has exhibited important roles in cell cycle progression, transcriptional activation, chromosome condensation, DNA repair and histone modification (5,7). An increasing number of studies have found that $V R K 1$ expression regulates the proliferation and survival of cells in normal or malignant tissues (7-9). During embryonic development of hematopoiesis, the development of the mouse liver is accompanied with high expression of VRK1. Similarly, high expression of VRK1 has been demonstrated in regenerated liver and liver cancer, which suggests that its expression is associated with the increase of the number of cells in the early hematopoietic process (10). In addition, VRK1 is highly expressed in high-proliferating cells, such as those found in the testis, thymus and fetal liver (6). Notably, a previous study has shown that $V R K 1$ induces the $\mathrm{G}_{1} / \mathrm{S}$ transition by promoting the expression of cyclin DI (CCNDI) at the $\mathrm{G}_{1} / \mathrm{S}$ phase (11). VRK1 expression has been shown to be upregulated in several types of cancer, including glioma, lung carcinomas, hepatocellular carcinoma, breast carcinomas and head and neck squamous cell carcinoma (9,12-14).

Barrier-to-autointegration factor 1 (BANF1) is encoded by the $B A N F 1$ gene and is a small, highly conserved DNA-binding protein of $10 \mathrm{kDa}$ in size that is located in the cytoplasm and nuclei of cells (15). BANF1 serves a crucial role in mitotic nuclear recombination, regulation of the stability of the pre-integration complex of retroviruses and in the regulation of transcriptional function (16). Margalit et al (15) 
reported a linkage of genomic DNA with the nuclear envelope in the interphase of mitosis through interactions with the nuclear envelope components (lamin) and BANF1 protein. Previous studies have also reported that phosphorylation regulates the DNA binding activity of BANF1 and its subcellular localization and dimerization $(17,18)$. It is important to note that Ser-4 is a major phosphorylation site of BANF1 during both the interphase and the mitotic phase (19). The phosphorylation of Ser-4 abrogates the interaction of BANF1 with DNA and reduces its interaction with the LEM domain and thereby disrupts the connection between the DNA and the nuclear envelope, which in turn maintains the normal process of the cell cycle (18). Previous studies $(7,20,21)$ have shown that VRK1 can catalyze the phosphorylation of $B A N F 1$, which is a high affinity substrate for $V R K 1$ protein kinase (22). Nichols et al (22) demonstrated that VRK1 regulated the interaction between BANF1 and DNA by phosphorylation of the N-terminus of BANF1. VRK1 participated directly in the regulation of the binding of chromatin to membrane proteins and BANF1 by facilitating the phosphorylation of the latter $(7,17)$. Results from the aforementioned studies led to the aim of the present study, which was to investigate the interaction of VRKI and BANFI and its association with the physiology of ESCC cancer cells.

$V R K 1$ and $B A N F 1$ expression levels were found to be elevated in ESCC tissues compared with the corresponding levels noted in adjacent non-tumor tissues. In addition, the expression levels of $V R K 1$ and $B A N F 1$ were significantly associated with the clinical characteristics of patients with esophageal cancer (23). In the present study, the ESCC cell lines EC109 and EC1 were used to examine the interaction between $V R K 1$ and $B A N F 1$ in ESCC. Small interfering (si) RNA was utilized to downregulate the expression of $V R K 1$ and the changes in the expression levels of BANF1 were investigated in ESCC cells. In addition, changes in proliferation and migration of ESCC cells were assessed to explore the potential of this protein in targeted therapy of ESCC. Taken collectively, the evidence in the present study indicated that $V R K 1$ and $B A N F 1$ may have pivotal roles during ESCC development and progression, and represent potential targets for novel ESCC treatments.

\section{Materials and methods}

Cell lines and cell culture. The human ESCC cell lines EC109 and EC1 were purchased from Sangon Biotech Co., Ltd. The cell lines were cultured and maintained in RPMI-1640 (Sangon Biotech Co., Ltd.) supplemented with $10 \%$ fetal bovine serum (Sangon Biotech Co., Ltd) at $37^{\circ} \mathrm{C}$ in the presence of $5 \% \mathrm{CO}_{2}$.

Cell transfection. The siRNA sequences targeting VRKl were constructed by Guangzhou RiboBio Co., Ltd. A total of three siRNA sequences were designed against $V R K 1$, and the efficacy of transfection was assessed using western blot analysis. The candidate VRK1 siRNA sequences were as follows: VRK1-176 (5'-GCAGUUGGAGAGAUA AUA ATT-3'), VRK1-571 (5'-GCAGCUAAGCUUAAGAAUUTT-3') and VRK1-862 (5'-CCAAUGGCUUACUGGCCAUTT-3'). The negative control siRNA (siNC) sequence was 5'-UUCUCC
GAACGUGUCACGUTT-3'. Downregulation of $V R K 1$ by siRNA in EC109 and EC1 cells was performed using the riboFECT ${ }^{\mathrm{TM}}$ CP Reagent (Guangzhou RiboBio Co. Ltd.,) according to the manufacturer's instructions. Cells underwent transfection when in the logarithmic growth phase. EC109 and $\mathrm{EC} 1$ cells were cultured in 6-well plates at a density of $4 \times 10^{5}$ cells/well at $37^{\circ} \mathrm{C}$, in the presence of $5 \% \mathrm{CO}_{2}$. The transfection was performed at $37^{\circ} \mathrm{C}$ for $24 \mathrm{~h}$ when the confluency of the cells was $\sim 30-50 \%$. The concentration of siRNAs transfected was $50 \mathrm{nM}$. Subsequent experimentation was performed $24 \mathrm{~h}$ after transfection.

Western blot analysis. The protein expression levels of VRK1 and $B A N F 1$ were detected by western blotting in ESCC cells following siRNA transfection. Transfected EC109 and EC1 cells were collected following transfection which lasted $24 \mathrm{~h}$ and washed three times with ice-cold PBS. The proteins were extracted using RIPA lysis buffer (Wuhan Boster Biological Technology, Ltd.) with PMSF (Boster) and the extracts were centrifuged at $4^{\circ} \mathrm{C}$, at $12,000 \mathrm{x}$ for $15 \mathrm{~min}$. The total protein concentration was determined using the BCA Protein Assay kit (Boster). Following denaturation of the proteins by boiling for $10 \mathrm{~min}$, equal amount of protein samples $(25 \mu \mathrm{g})$ were separated by $10 \%$ SDS-PAGE (Boster) and subsequently transferred to PVDF membranes (Boster). Subsequently, the membranes were blocked with TBS + Tween 20 (TBST; 0.1\% Tween-20) containing 5\% non-fat milk (Boster) at room temperature for $1 \mathrm{~h}$ and subsequently incubated with primary antibodies against VRK1 (1:1,000; cat. no. ab211358; Abcam), BANF1 (1:1,000; cat. no. ab231331; Abcam) and GAPDH (1:1,000; cat. no. ab9485; Abcam) overnight at $4^{\circ} \mathrm{C}$. The membranes were rinsed with TBST 3-5 times and incubated with horseradish peroxidase-conjugated secondary antibodies $(1: 1,000$; cat. no. bs-40295G-HRP; BIOSS) for $1 \mathrm{~h}$ at room temperature. Following an additional rinse with TBST, the protein bands were visualized using the eECL Western Blot Kit (Beyotime Institute of Biotechnology) and a UVP gel imaging scanning analyzer (GENE). Protein expression analysis was performed using Image J software v1.8.0 (National Institute of Health) to calculate the relative protein expression. GAPDH was used to normalize protein expression.

Cell proliferation assay. EC109 and EC1 cell proliferation was examined by the Cell Counting Kit-8 (CCK-8; Sangon Biotech) assay. A total of $2 \times 10^{3}$ cells/well were seeded in 96 -well plates. In the following 3 days, $10 \mu \mathrm{l}$ CCK- 8 reagent was added in $100 \mu \mathrm{l}$ fresh medium after culturing for $12,24,36,48,60$ and $72 \mathrm{~h}$ and the cells were incubated at $37^{\circ} \mathrm{C}$ for $2 \mathrm{~h}$. The absorbance was measured at $450 \mathrm{~nm}$ to calculate the number of viable cells. All the assays were repeated three times.

Flow cytometry and cell cycle analysis. Cells were harvested by trypsinization without EDTA and collected by centrifugation at $400 \mathrm{x} \mathrm{g}$ in $37^{\circ} \mathrm{C}$ for $5 \mathrm{~min}$. The cells were resuspended and washed twice with pre-cooled PBS to obtain a single-cell suspension. The cells were fixed overnight at $4^{\circ} \mathrm{C}$ with $500 \mu \mathrm{l}$ $70 \%$ alcohol. The cell suspension concentration was estimated at $1 \times 10^{6}$ cells $/ \mathrm{ml}$. Cell cycle analysis was performed using propidium iodide staining following treatment with RNaseA (cat. no. R1030; Beijing Solarbio Science \& Technology Co., 
A

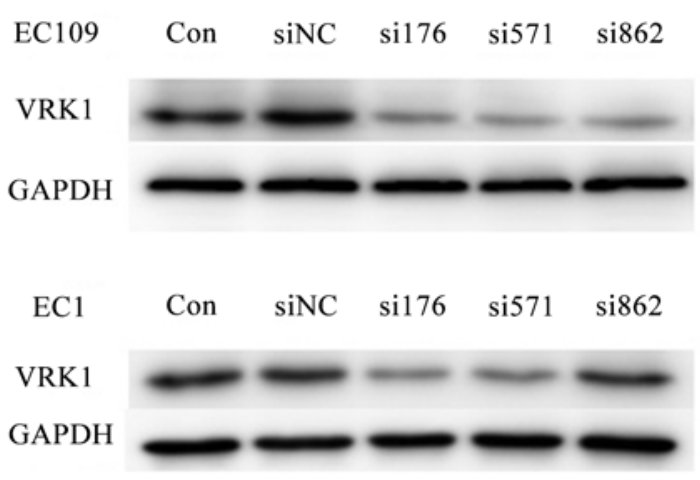

$\mathrm{B}$

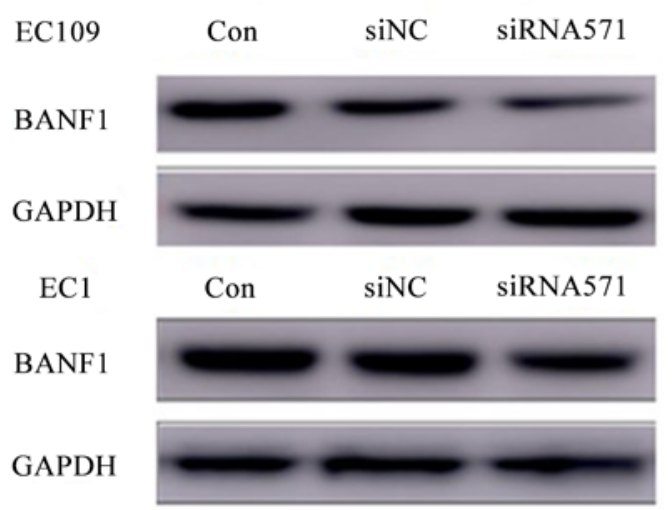

$\mathrm{C}$

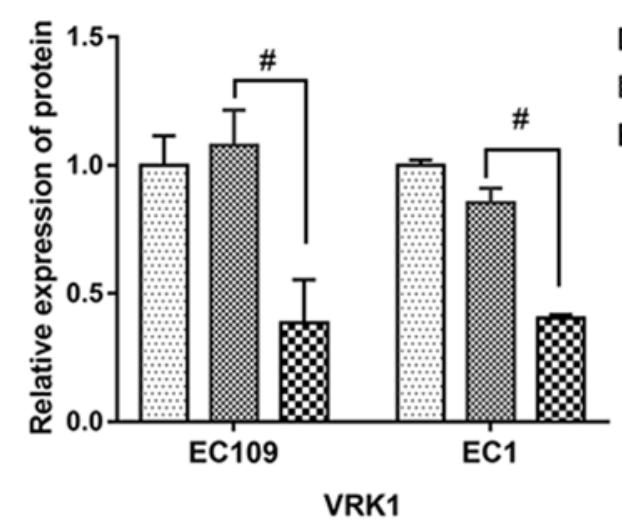

$\mathrm{D}$
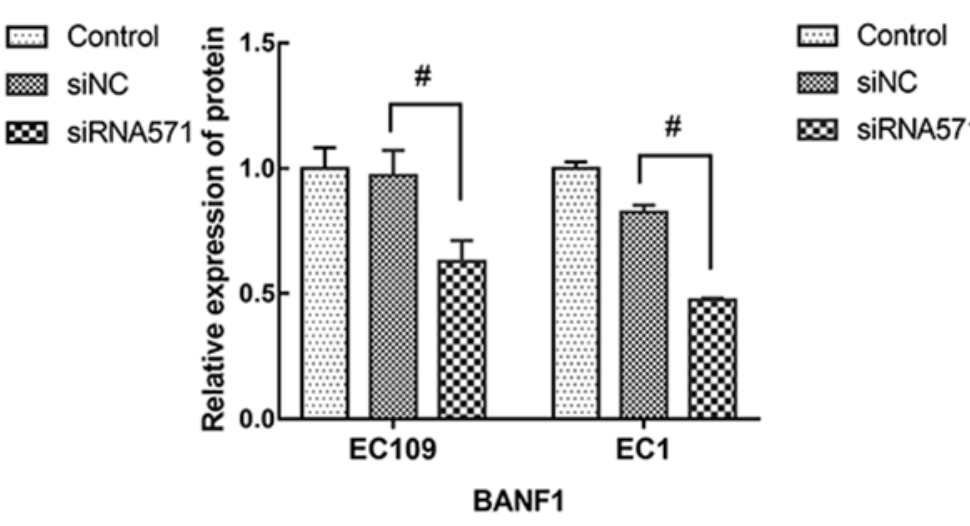

Figure 1. BANF1 expression is reduced following inhibition of the expression of VRK1 in ESCC cell lines EC109 and EC1. (A) Expression of VRK1 following transfection with gene-specific siRNAs, including VRK1-176, VRK1-571 and VRK1-862 in ESCC cell lines EC109 and EC1. In order to select a siRNA with stable transfection effect and eliminate the interference of other factors, 2 siRNAs (VRK1-176 and VRK1-571) were selected for a preliminary experiment. As the status of ESCC cells transfected with siRNA-176 was unstable, VRK1-571 was selected. (B) Expression of BANF1 was reduced after the expression of VRK1 was inhibited, as shown by western blot. (C) Semi-quantification analysis of the expression of VRK1 and BANF1 after transfection with VRK1-siRNA571 from (B). (D) Semi-quantification analysis of the expression of BANF1 after transfection with VRK1-siRNA571 from (B). ${ }^{"} \mathrm{P}<0.05$. BANF1, barrier-to-autointegration factor 1; con, non-transfected control; ESCC, esophageal squamous cell carcinoma; NC, negative control; siRNA, small interfering RNA; VRK1, vaccinia-related kinase.

Ltd.) to remove contaminating RNA. The contents of DNA in each phase $\left(G_{0} / G_{1}, S\right.$ and $\left.G_{2} / M\right)$ were detected using a FACSAria flow cytometer (BD Biosciences) and ModFit LT software v.4.0 (Verity Software House, Inc.).

Transwell migration assays. The Transwell migration assay was performed to detect the changes of the migratory ability of ESCC cells following transfection with siRNAs. The migration assay was performed by a Transwell system $(8.0 \mu \mathrm{m}$ pore size; 24-well insert). The cells were seeded at a density of $5 \times 10^{4}$ cells in FBS-free DMEM (Sangon Biotech Co., Ltd) to the permeable membrane of the insert in the upper chamber. The lower chamber was filled with $600 \mu 1$ DMEM containing $10 \%$ FBS (Sangon Biotech Co., Ltd). Following incubation for $24 \mathrm{~h}$ at $37^{\circ} \mathrm{C}$, the cells left on the upper surface of the insert were carefully removed with a cotton swab and the migrated tumor cells on the lower surface were stained by crystal violet for cell number determination.

Statistical analysis. All experimental data were analyzed using the SPSS software package, v21.0 (IBM Corp.). The GraphPad Prism software (v6.0; GraphPad Software, Inc.) was used for graph preparation. Data were analyzed by Student's t-test, $\chi^{2}$ or ANOVA for multiple groups followed by a Bonferroni's post hoc test. The results are expressed as the mean \pm standard deviation, and $\mathrm{P}<0.05$ was considered to indicate a statistically significant difference.

\section{Results}

Depletion of VRK1 results in downregulation of $B A N F 1$ expression. A total of three siRNA sequences including VRK1-176, VRK1-571 and VRK1-862 were specifically designed and synthesized for transfection into EC109 and EC1 cells, respectively. Following culture for 24 h, siVRK1-571 exhibited the highest reduction efficiency of $V R K 1$ protein expression and was selected as the siRNA to be used in the subsequent experiments (Fig. 1A). Western blot analysis indicated no significant difference in $V R K 1$ protein expression between the blank control group and the siNC-transfected group of the EC109 and EC1 cell lines (Fig. 1C). However, the expression levels of VRK1 in the siVRK1-571 interference group were significantly lower compared with those in the siNC group, with a decrease of 62.40 and $52.14 \%$ in the 

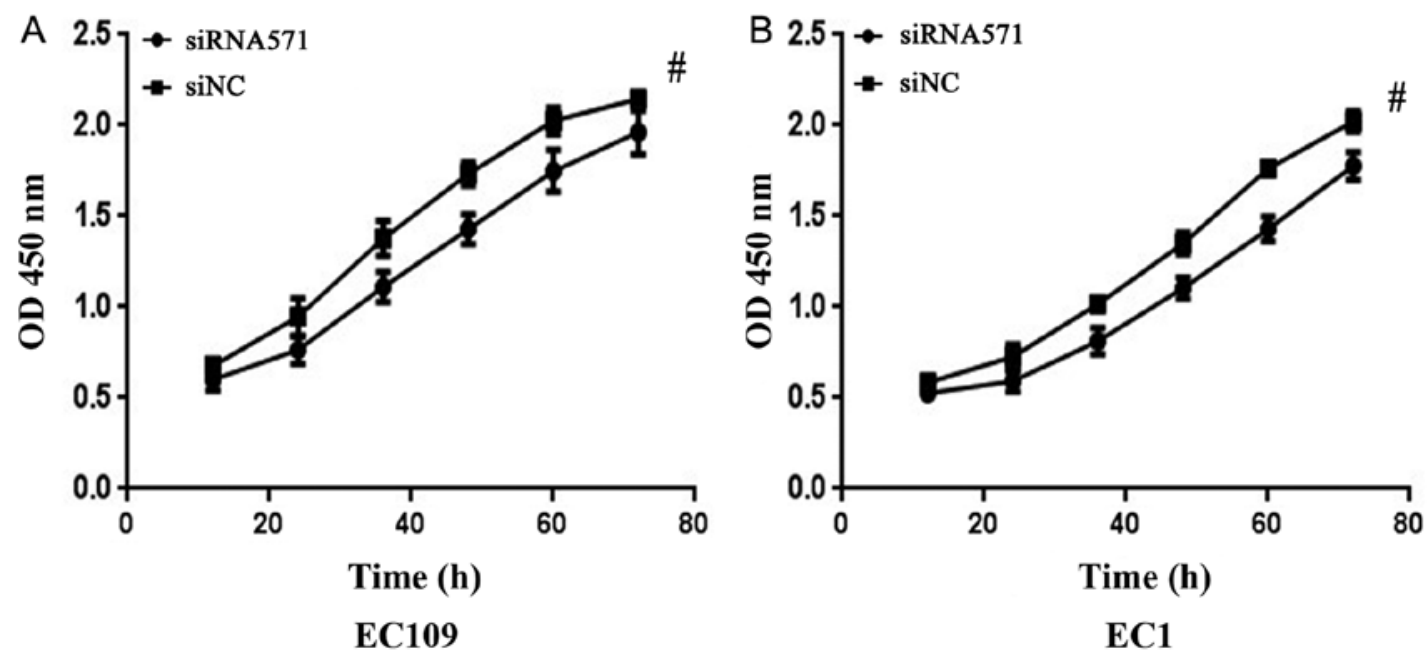

Figure 2. Proliferation of ESCC cells detected via CCK-8 assay. Growth curves of (A) EC109 and (B) EC1 cells transfected with either siNC or siRNA571. ${ }^{\text {"P }}<0.05$ vs. siNC. NC, negative control; siRNA, small interfering RNA.

EC109 and EC1 cell lines, respectively $(\mathrm{P}<0.05)$. These results demonstrated that the expression of $V R K 1$ was downregulated by siRNA specifically and effectively.

Furthermore, western blot analysis demonstrated that the protein expression levels of $B A N F 1$ in siVRK1-571-transfected cells were significantly lower compared with those of the siNC-transfected control group, with a decrease of 24.51 and $52.87 \%$ in the EC109 and EC1 cell lines, respectively $(\mathrm{P}<0.05$; Fig. 1B and $\mathrm{C})$. In contrast to these findings, BANF1 protein expression levels did not reveal a significant difference between the blank and the negative control groups.

Depletion of VRK1 suppresses the proliferation of ESCC cells. CCK-8 cell proliferation analysis was conducted on the EC109 and $\mathrm{EC} 1$ cell lines to investigate the effects of $V R K 1$ on the proliferation of ESCC cells. Following $12 \mathrm{~h}$ of transfection, the cells proliferated less effectively and the inhibition rate of EC109 and EC1 cells reached the maximum effect at 48 and $60 \mathrm{~h}$ after transfection, respectively $(\mathrm{P}<0.05$; Fig. 2). The results demonstrated that downregulation of $V R K 1$ significantly inhibited the proliferative ability of EC109 and EC1 cells.

Depletion of VRK1 triggers cell cycle arrest in ESCC cells. To further verify the effect of $V R K I$ on ESCC cell proliferation, $V R K I$ was depleted and the effects on cell cycle of ESCC cells were examined using flow cytometry. Flow cytometric analysis of EC109 cells indicated that depletion of VRK1 led to a significantly increased population of cells in the $\mathrm{S}$ phase compared with that noted in the negative control group ( $\mathrm{P}=0.003$; Fig. 3). The percentage of cells treated with siVRK1-571 at the $G_{0} / G_{1}$ and $\mathrm{G}_{2} / \mathrm{M}$ phases was significantly lower compared with that noted in the siNC group $\left(\mathrm{P}_{\mathrm{G} 0 / \mathrm{G} 1}=0.005 ; \mathrm{P}_{\mathrm{G} 2 / \mathrm{M}}=0.001\right)$. Similarly, in EC1 cells, the percentage of cells treated with siVRK1-571 at the $\mathrm{G}_{2} / \mathrm{M}$ phase was significantly lower compared with that in the negative control group $(\mathrm{P}=0.022)$. The percentage of cells treated with siVRK1-571 at the S phase of the cell cycle was higher than that noted in the negative control group $(\mathrm{P}=0.023$; Fig. 3). The results further demonstrated that depletion of VRKI induced cell cycle arrest at the $\mathrm{S}$ phase, which in turn resulted in inhibition of cell proliferation of ESCC cells.
Depletion of VRK1 inhibits ESCC cell migration. Metastasis is a critical problem during cancer therapy. Therefore, the Transwell assay was used to assess whether loss of VRKI could affect tumor migratory activity. The results demonstrated that siRNA-based depletion of VRK1 significantly reduced the number of migrating cells compared with that noted in the blank control (CON) and the negative control (siNC) groups (Fig. 4). These results indicated that $V R K 1$ may serve an essential role in the migration of ESCC.

\section{Discussion}

Previous reports suggested that $V R K 1$ has a pivotal role in the regulation of a variety of cellular physiological activities; in various cancers, the expression levels of VRK1 have been significantly associated with cancer progression and prognosis $(9,12,13,24)$. In addition, $B A N F 1$ has been proven to be a valuable member in regulating the reassembly of the nuclear envelope and maintaining appropriate nuclear architecture during mitosis (15). Certain studies $(20,21)$ have suggested that $B A N F 1$ is an efficient substrate for $V R K 1$ as $V R K 1$ phosphorylates the N-terminus of $B A N F 1$ (22). These findings suggested that $V R K 1$ and $B A N F 1$ were closely associated with cell cycle regulation, which led to the hypothesis that the expression of BANF1 and VRK1 may contribute to the development of ESCC. This hypothesis was examined in the present study to provide additional information with regard to the molecular mechanisms of action during ESCC pathogenesis.

In a previous study conducted by our group, the mRNA and protein expression levels of VRK1 and BANF1 were higher in tumor tissues compared with those noted in the adjacent non-cancerous tissues, as determined by RT-qPCR and immunohistochemical analyses (23). Furthermore, the expression levels of $V R K 1$ and $B A N F 1$ were associated with the tumor, node, metastasis (TNM) stage and the differentiation degree of ESCC patients (23). In the present study, VRK1 expression was depleted by siRNA in ESCC cell lines and, consequently, the expression levels of BANFI were significantly downregulated. In addition, in the present study it was demonstrated that the proliferative activity of EC109 and EC1 


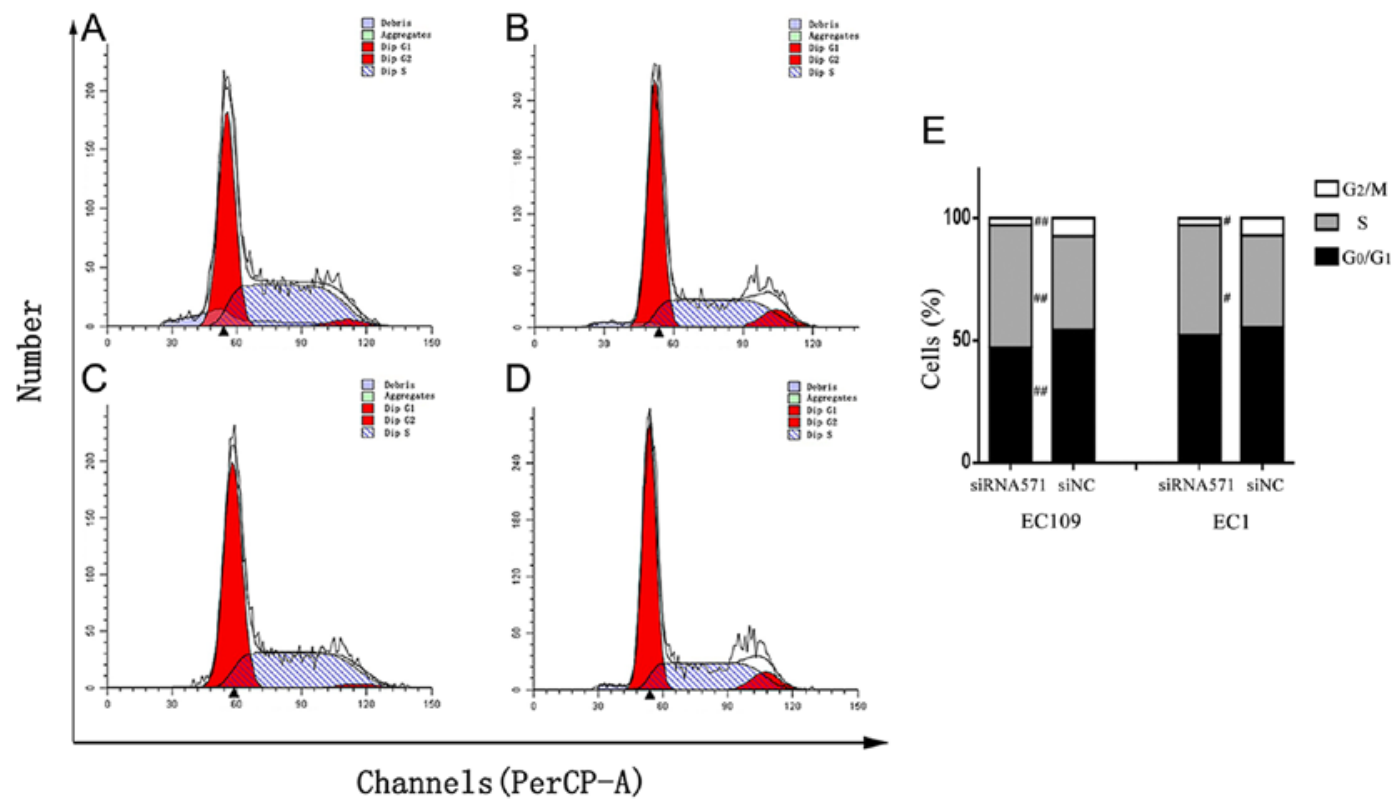

Figure 3. Cell cycle assay. Cell cycle distribution of EC109 and EC1 cells was determined by flow cytometry. (A) EC109-siRNA571, (B) EC109-siNC, (C) EC1siRNA571 and (D) EC1-siNC, (E) Cell cycle percentage histogram of the G2/M, S and G0/G1 phases of EC109 and EC1 cells. ${ }^{\#} \mathrm{P}<0.05$ and ${ }^{\# \#} \mathrm{P}<0.01 \mathrm{vs}$. siNC. NC, negative control; si, small interfering RNA; VRK1, vaccinia-related kinase 1.

cells were significantly reduced following inhibition of VRKI expression. Flow cytometric analysis further revealed that the cell cycle was inhibited mainly at the $\mathrm{S}$ phase. Moreover, Transwell assays indicated that VRK1 depletion significantly suppressed the migratory ability of ESCC cells. In conclusion, depletion of $V R K 1$ resulted in the downregulation of BANF1 and suppressed the proliferation and migration of ESCC cells.

A growing body of evidence indicates that $V R K 1$ has an important role in the development of tumors. In hepatocellular carcinoma, the expression levels of VRK1 in cancer tissues were higher than those noted in normal and adjacent tissues (12). In breast cancer, although high expression levels of VRK1 exhibited a protective effect on DNA damage, they also led to poorer disease prognosis (25). Therefore, VRK1 may represent a potential prognostic indicator for breast cancer $(24,25)$. Further experiments in mammary epithelial cells demonstrated that depletion of VRK1 inhibits their proliferation and metastasis in vitro and in vivo (8). VRKI has also been suggested as a proliferative marker in head and neck squamous cell carcinoma and as a potential drug target in breast cancer and lung adenocarcinomas $(9,14,25)$. In addition, $V R K 1$ has an important role in the stress response of DNA damage induced by ionizing radiation and ultraviolet radiation $(26,27)$. In the present study, the increased $V R K I$ levels in ESCC were consistent with the results noted in other types of cancer, such as hepatocellular carcinoma and breast cancer $(12,13)$. Therefore, the data suggested that VRK1 may contribute to the progression of the ESCC. In the present study, the CCK- 8 cell proliferation assay demonstrated that the proliferative activity was decreased in EC109 and EC1 cells following depletion of the expression of VRK1. Moreover, Transwell experiments indicated that the migratory ability of ESCC cells was also reduced. Given that the reduction of $V R K 1$ expression could inhibit the proliferation and migration of ESCC cells, it is hypothesized that VRKI could represent a potent new target for the treatment of esophageal cancer.

$B A N F 1$ has been shown to bind to double-stranded DNA at the LEM domain of transcriptional regulators and at the histone H3 protein, which is localized in the nucleus (16). Previous findings suggested that lamin, LEM-domain proteins and $B A N F 1$ performed essential functions in chromatin organization and cell division (28). Lamin-A/C, lamin-associated polypeptide $2 \alpha$ and $B A N F 1$ proteins constitute protein complexes that regulate mitotic spindle assembly and localization during mitosis (29). In addition, BANF1 was directly involved in the formation of the nuclear envelope (NE). These results revealed the essential role of $B A N F 1$ in regulating the mitotic process and normal cell cycle progression. Moreover, $B A N F 1$ has been reported to be a novel biomarker for gastric cancer (30). In one of our previous studies, it was shown that $B A N F 1$ was highly expressed in ESCC and that its expression was associated with the TNM stage and the tumor differentiation degree of ESCC patients (23). The data suggested that the highly expressed levels of BANF1 may lead to an abnormal mitotic process of ESCC cells through regulation of the interaction of DNA with perinuclear proteins and the assembly and localization of the spindle filaments.

Notably, BANF1 has also been established as a high-affinity substrate of $V R K 1$. In addition, it has been shown that depletion of VRK1 affects the interaction between BANF1 and DNA, thereby affecting nuclear membrane structure and mitotic chromosomal dynamics (31). Together, these studies suggested that $V R K 1$ and $B A N F 1$ were closely associated with the regulation of normal mitosis. The essential role of $V R K 1$ and $B A N F 1$ in regulating the mitotic nuclear reassembly indicates that the abnormal expression of these two proteins in ESCC may affect the normal cellular functions, such as nuclear organization and cell cycle progression. Based on the high expression of VRK1 and $B A N F 1$ in ESCC, the present study demonstrated that the 
A
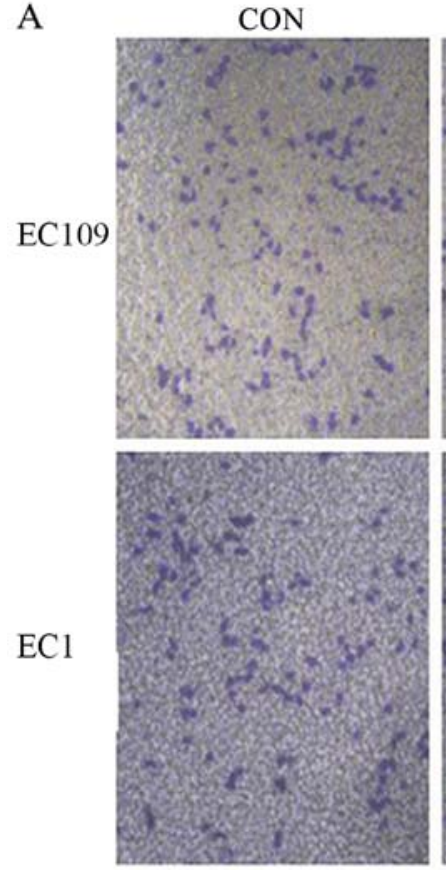

siNC
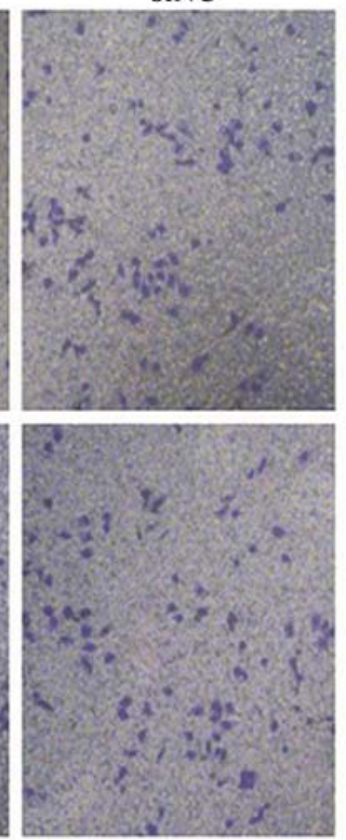

siRNA571
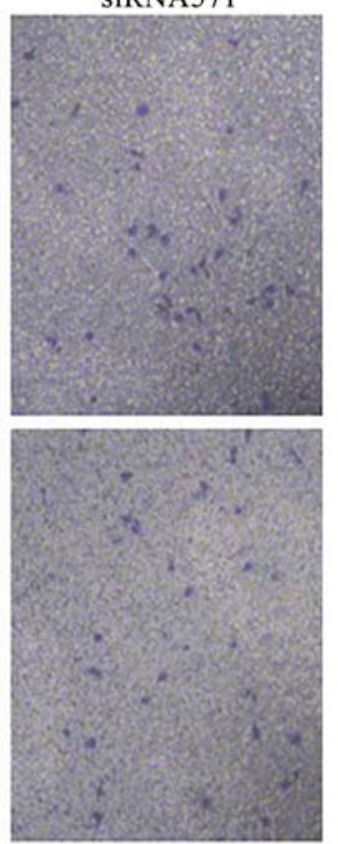

$\mathrm{B}$

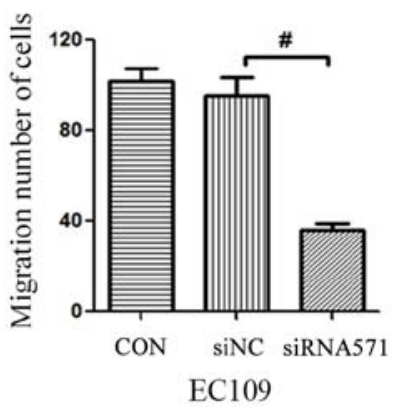

$\mathrm{C}$

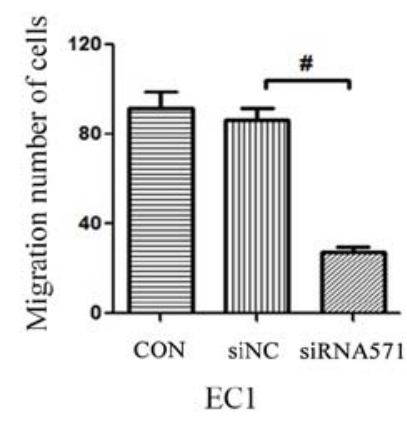

Figure 4. Transwell migration assays. (A) Migratory abilities of EC109 and EC1 cells following transfection and cell culture for $12 \mathrm{~h}$. Comparison of the migratory abilities of (B) EC109 and (C) EC1 cells among different groups; magnification x200. ${ }^{\text {P }}<0.05$. siNC, siRNA negative control; siRNA571, siRNA targeting VRK1-571.

$B A N F 1$ protein was downregulated following depletion of the expression of $V R K 1$ in ESCC cells. These findings indicated a putative interaction between $V R K 1$ and $B A N F 1$ in promoting the development of ESCC.

The present study data indicated that the depletion of $V R K 1$ and the subsequent downregulation of $B A N F 1$ resulted in changes in the cell cycle distribution, which mainly manifested as arrest of the cell cycle at the $S$ phase. The results indicated that the depletion of VRK1 may affect cell proliferation by blocking cell cycle progression. These results support previous studies $(9,11,12)$. Previous research in head and neck squamous cell carcinoma suggested that VRK1 plays a role in cell cycle regulation and may be a new control mechanism of cell cycle, particularly late in G1-S phase (9). In addition, Valbuena et al (11) demonstrated that the elimination of VRK1 by siRNA results in a G1 block in cell division. As a member of the novel $V R K$ protein family, $V R K 1$ can phosphorylate the Thr-18 region of $p 53$, a vital tumor suppressor protein (32). The region that becomes phosphorylated comprises the $M D M-2$ binding domain and is required for maintaining $p 53$ stability (33). VRK1 has been shown to be a key regulator of $p 53$ and to control cell proliferation. A study by Waters et al (34) further revealed that $V R K 1$ promoted germ cell proliferation by preventing $p 53$ from triggering abnormal cell cycle arrest. An additional study reported a newly formed autoregulatory loop between $p 53$ and $V R K 1$ (35). Therefore, $V R K 1$ has been regarded as an upstream regulator of $p 53$, which participates in the integration of various cell signals by $p 53$ (36). In addition, VRK1 can phosphorylate other transcription factors, such as $c$-Jun and $A T F$, which play essential roles in cell cycle regulation $(37,38)$. Valbuena et al (11) demonstrated that the loss of VRK1 led to the block of the cell cycle. Similar findings were presented in the current report highlighting that the downregulation of $V R K 1$ triggered cell cycle arrest at the $\mathrm{G}_{1}$ phase. In addition, $V R K 1$ is a regulator of cyclin D1 $(C C N D 1)$ expression in the DNA replication period (39). $V R K 1$ is also known to phosphorylate histone $\mathrm{H} 3$ to regulate chromatin condensation $(40,41)$. Collectively, these results suggest that further study of the interaction between VRK1 and transcription factors such as p53 may be a meaningful direction for exploring the mechanism of cell cycle regulation in ESCC. However, further experiments are required to confirm this assumption. $B A N F 1$ was also reported to perform crucial functions in both the mitotic phase and the cell cycle interphase (42). The reduction or loss of BANF1 expression caused the aberrant cell cycle progression or phenotype. For example, BANF1-null Drosophila flies present various cell phenotypes that involve cell cycle arrest, chromatin clumping, abnormal lamin distribution and nuclear lamina structure (43). These findings indicated that the depletion of $B A N F 1$ could affect cell cycle progression. In the present study, $B A N F 1$ expression was decreased following depletion of the expression of $V R K 1$. Therefore, it was hypothesized that $V R K 1$ may regulate abnormal cell proliferation by affecting $B A N F 1$ expression, which may be a possible mechanism in the process of esophageal cancer development.

In conclusion, results from the present study indicated that downregulation of $V R K 1$ suppressed the proliferative and migratory ability of ESCC cells in vitro and suggested that $V R K 1$ may serve as a therapeutic target in the treatment of ESCC. Furthermore, VRK1 depletion suppressed BANF1 expression. Taken collectively, the aforementioned findings suggested a potential connection between $V R K 1$ and $B A N F 1$ in the development of ESCC. The results presented may be used to further examine the interaction between $V R K 1$ and $B A N F 1$ in the progression of ESCC. 


\section{Acknowledgements}

The authors would like to thank Professor Chunfeng Ren (First Affiliated Hospital of Zhengzhou University) for providing laboratory support.

\section{Funding}

The work was supported by The Henan Provincial Department of Education Key Science and Technology Project (grant no. 18A320007) and The Ministry of Health and Welfare Committee (grant no. SBGJ2018013).

\section{Availability of data and materials}

The datasets used and/or analyzed during the present study are available from the corresponding author on reasonable request.

\section{Authors' contributions}

ZR, JG, CX and XL performed the experiments. ZR wrote the manuscript. ZR, YL and JL analyzed and interpreted the data and revised the manuscript for important intellectual content. HL participated in the design of the research, and was responsible for the guidance in the experimental process and gave the approval for the final version of the manuscript. All authors have read and approved the final manuscript.

\section{Ethics approval and consent to participate}

Not applicable

\section{Patient consent for publication}

Not applicable.

\section{Competing interests}

The authors declare that they have no competing interests.

\section{References}

1. Chen W, Zheng R, Baade PD, Zhang S, Zeng H, Bray F, Jemal A, Yu XQ and He J: Cancer statistics in China, 2015. CA Cancer J Clin 66: 115-132, 2016.

2. Bray F, Ferlay J, Soerjomataram I, Siegel RL, Torre LA and Jemal A: Global cancer statistics 2018: GLOBOCAN estimates of incidence and mortality worldwide for 36 cancers in 185 countries. CA Cancer J Clin 68: 394-424, 2018.

3. Enzinger PC and Mayer RJ: Esophageal cancer. N Engl J Med 349: 2241-2252, 2003

4. Wu PC and Posner MC: The role of surgery in the management of oesophageal cancer. Lancet Oncol 4: 481-488, 2003.

5. Nichols RJ and Traktman P: Characterization of three paralogous members of the mammalian vaccinia related kinase family. J Biol Chem 279: 7934-7946, 2004

6. Nezu J, Oku A, Jones MH and Shimane M: Identification of two novel human putative serine/threonine kinases, VRK1 and VRK2, with structural similarity to vaccinia virus B1R kinase. Genomics 45: 327-331, 1997.

7. Valbuena A, Sanz-García M, López-Sánchez I, Vega FM and Lazo PA: Roles of VRK1 as a new player in the control of biological processes required for cell division. Cell Signal 23: 1267-1272, 2011.

8. Molitor TP and Traktman P: Molecular genetic analysis of VRK1 in mammary epithelial cells: Depletion slows proliferation in vitro and tumor growth and metastasis in vivo. Oncogenesis 2: e48, 2013.
9. Santos CR, Rodríguez-Pinilla M, VegaFM,Rodríguez-Peralto JL, Blanco S, Sevilla A, Valbuena A, Hernández T, van Wijnen AJ, Li F, et al: VRK1 signaling pathway in the context of the proliferation phenotype in head and neck squamous cell carcinoma. Mol Cancer Res 4: 177-185, 2006.

10. Vega FM, Gonzalo P, Gaspar ML and Lazo PA: Expression of the VRK (vaccinia-related kinase) gene family of p53 regulators in murine hematopoietic development. FEBS Lett 544: 176-180, 2003.

11. Valbuena A, López-Sánchez I and Lazo PA: Human VRK1 is an early response gene and its loss causes a block in cell cycle progression. PLoS One 3: e1642, 2008.

12. Lee N, Kwon JH, Kim YB, Kim SH, Park SJ, Xu W, Jung HY, Kim KT, Wang HJ and Choi KY: Vaccinia-related kinase 1 promotes hepatocellular carcinoma by controlling the levels of cell cycle regulators associated with G1/S transition. Oncotarget 6: 30130-30148, 2015.

13. Mon AM, MacKinnon AC Jr and Traktman P: Overexpression of the VRK1 kinase, which is associated with breast cancer, induces a mesenchymal to epithelial transition in mammary epithelial cells. PLoS One 13: e0203397, 2018.

14. Kim IJ, Quigley D, To MD, Pham P, Lin K, Jo B, Jen KY, Raz D, KimJ,MaoJH,etal:Rewiring of humanlung celllineageandmitotic networks in lung adenocarcinomas. Nat Commun 4: 1701, 2013.

15. Margalit A, Brachner A, Gotzmann J, Foisner R and Gruenbaum Y: Barrier-to-autointegration factor-a BAFfling little protein. Trends Cell Biol 17: 202-208, 2007.

16. Margalit A, Neufeld E, Feinstein N, Wilson KL, Podbilewicz B and Gruenbaum Y: Barrier to autointegration factor blocks premature cell fusion and maintains adult muscle integrity in $\mathrm{C}$. elegans. J Cell Biol 178: 661-673, 2007.

17. Gorjanacz M, Klerkx EP, Galy V, Santarella R, López-Iglesias C, Askjaer P and Mattaj IW: Caenorhabditis elegans BAF-1 and its kinase VRK-1 participate directly in post-mitotic nuclear envelope assembly. EMBO J 26: 132-143, 2007.

18. Jamin A, Wicklund A and Wiebe MS: Cell- and virus-mediated regulation of the barrier-to-autointegration factor's phosphorylation state controls its DNA binding, dimerization, subcellular localization, and antipox viral activity. J Virol 88: 5342-5355, 2014.

19. Bengtsson L and Wilson KL: Barrier-to-autointegration factor phosphorylation on ser- 4 regulates emerin binding to lamin A in vitro and emerin localization in vivo. Mol Biol Cell 17: 1154-1163, 2006.

20. Lancaster OM, Cullen CF and Ohkura H: NHK-1 phosphorylates BAF to allow karyosome formation in the drosophila oocyte nucleus. J Cell Biol 179: 817-824, 2007.

21. Kim W, Lyu HN, Kwon HS, Kim YS, Lee KH, Kim DY, Chakraborty G, Choi KY, Yoon HS and Kim KT: Obtusilactone $\mathrm{B}$ from machilus thunbergii targets barrier-to-autointegration factor to treat cancer. Mol Pharmacol 83: 367-376, 2013.

22. Nichols RJ, Wiebe MS and Traktman P: The vaccinia-related kinases phosphorylate the $\mathrm{N}^{\prime}$ terminus of $\mathrm{BAF}$, regulating its interaction with DNA and its retention in the nucleus. Mol Biol Cell 17: 2451-2464, 2006

23. Li J, Wang T, Pei L, Jing J, Hu W, Sun T and Liu H: Expression of VRK1 and the downstream gene BANF1 in esophageal cancer. Biomed Pharmacother 89: 1086-1091, 2017.

24. Martin KJ, Patrick DR, Bissell MJ and Fournier MV: Prognostic breast cancer signature identified from 3D culture model accurately predicts clinical outcome across independent datasets. PLoS One 3: e2994, 2008.

25. Salzano M, Vázquez-Cedeira M, Sanz-García M, Valbuena A, Blanco S, Fernández IF and Lazo PA: Vaccinia-Related kinase 1 (VRK1) confers resistance to DNA-damaging agents in human breast cancer by affecting DNA damage response. Oncotarget 5: 1770-1778, 2014

26. Sanz-García M, Monsalve DM, Sevilla A and Lazo PA: Vaccinia-Related kinase 1 (VRK1) is an upstream nucleosomal kinase required for the assembly of 53BP1 foci in response to ionizing radiation-induced DNA damage. J Biol Chem 287: 23757-23768, 2012.

27. López-Sánchez I, Valbuena A, Vázquez-Cedeira M, Khadake J, Sanz-García M, Carrillo-Jiménez A and Lazo PA: VRK1 interacts with $\mathrm{p} 53$ forming a basal complex that is activated by UV-induced DNA damage. FEBS Lett 588: 692-700, 2014.

28. Margalit A, Liu J, Fridkin A, Wilson KL and Gruenbaum Y: A lamin-dependent pathway that regulates nuclear organization, cell cycle progression and germ cell development. Novartis Found Symp 264: 231-240, 2005.

29. Qi R, Xu N, Wang G, Ren H, Li S, Lei J, Lin Q, Wang L, Gu X, Zhang $\mathrm{H}$, et al: The lamin-A/C-LAP2alpha-BAF1 protein complex regulates mitotic spindle assembly and positioning. J Cell Sci 128: 2830-2841, 2015. 
30. Li J, Hu B, Fang L, Gao Y, Shi S, He H, Liu X and Yuan C: Barrier-To-Autointegration factor 1: A novel biomarker for gastric cancer. Oncol Lett 16: 6488-6494, 2018.

31. Molitor TP and Traktman P: Depletion of the protein kinase VRK1 disrupts nuclear envelope morphology and leads to BAF retention on mitotic chromosomes. Mol Biol Cell 25: 891-903, 2014.

32. Reinhardt HC and Schumacher B: The p53 network: Cellular and systemic DNA damage responses in aging and cancer. Trends Genet 28: 128-136, 2012.

33. Barcia R, López-Borges S, Vega FM and Lazo PA: Kinetic properties of p53 phosphorylation by the human vaccinia-related kinase 1. Arch Biochem Biophys 399: 1-5, 2002.

34. Waters K, Yang AZ and Reinke V: Genome-Wide analysis of germ cell proliferation in C.elegans identifies VRK-1 as a key regulator of CEP-1/p53. Dev Biol 344: 1011-1025, 2010.

35. Valbuena A, Vega FM, Blanco S and Lazo PA: P53 downregulates its activating vaccinia-related kinase 1 , forming a new autoregulatory loop. Mol Cell Biol 26: 4782-4793, 2006.

36. Lopez-Borges S and Lazo PA: The human vaccinia-related kinase 1 (VRK1) phosphorylates threonine-18 within the mdm-2 binding site of the p53 tumour suppressor protein. Oncogene 19: 3656-3664, 2000.

37. Sevilla A, Santos CR, Barcia R, Vega FM and Lazo PA: C-Jun phosphorylation by the human vaccinia-related kinase 1 (VRK1) and its cooperation with the N-terminal kinase of c-Jun (JNK) Oncogene 23: 8950-8958, 2004.

38. Sevilla A, Santos CR, Vega FM and Lazo PA: Human vaccinia-related kinase 1 (VRK1) activates the ATF2 transcriptional activity by novel phosphorylation on Thr-73 and Ser-62 and cooperates with JNK. J Biol Chem 279: 27458-27465, 2004.
39. Kang TH, Park DY, Kim W and Kim KT: VRK1 phosphorylates CREB and mediates CCND1 expression. J Cell Sci 121: 3035-3041, 2008.

40. Shin J, Chakraborty G, Bharatham N, Kang C, Tochio N, Koshiba S, Kigawa T, Kim W, Kim KT and Yoon HS: NMR solution structure of human vaccinia-related kinase 1 (VRK1) reveals the C-terminal tail essential for its structural stability and autocatalytic activity. J Biol Chem 286: 22131-22138, 2011.

41. Kim W, Chakraborty G, Kim S, Shin J, Park CH, Jeong MW, Bharatham N, Yoon HS and Kim KT: Macro histone H2A1.2 (macroH2A1) protein suppresses mitotic kinase VRK1 during interphase. J Biol Chem 287: 5278-5289, 2012.

42. Cox JL, Mallanna SK, Ormsbee BD, Desler M, Wiebe MS and Rizzino A: Banf1 is required to maintain the self-renewal of both mouse and human embryonic stem cells. J Cell Sci 124: 2654-2665, 2011.

43. Furukawa K, Sugiyama S, Osouda S, Goto H, Inagaki M, Horigome T, Omata S, McConnell M, Fisher PA and Nishida Y: Barrier-to-autointegration factor plays crucial roles in cell cycle progression and nuclear organization in drosophila. J Cell Sci 116: 3811-3823, 2003. 